\title{
ON THE CONSTRUCTION OF SPLIT-FACE TOPOLOGIES
}

\author{
BY \\ ALAN GLEIT(1)
}

\begin{abstract}
We give a general theorem to facilitate the construction of interesting examples of split-face topologies of compact, convex sets.
\end{abstract}

Introduction. Let $K$ be a compact convex set in a locally convex topological vector space. Let $F$ be a closed face of $K$ and $F^{\prime}$ be the union of all faces of $K$ disjoint from $F$. It is always true that $K=\operatorname{co}\left(F \cup F^{\prime}\right)$ [1, Proposition II.6.5]. The face $F$ is said to be split if $F^{\prime}$ is a face and $K$ is the direct convex sum of $F$ and $F^{\prime}[1$, p. 133], i.e. if each $x \in K$ can be expressed by a unique convex combination

$$
x=\lambda y+(1-\lambda) z
$$

with $0 \leq \lambda \leq 1, y \in F$ and $z \in F^{\prime}$. The collection

$$
\{F \cap \text { extreme points of } K \mid F \text { closed split face of } K\}
$$

forms the closed sets for a topology on the extreme points of $K$ called the splitface or facial topology [1, p. 143]. Much is known about the split-face topology but there is a distinct lack of many interesting examples. This paper provides the first general results which may help to alleviate this problem.

We are much indebted to $\mathrm{P}$. Taylor whose example (reproduced below) started us in the right direction.

Throughout, for a set $D \subseteq Y$ we let $D^{C}$ be the complement of $D$ in $Y$.

The construction. Let $Y$ be a compact Hausdorff space and $X$ a closed subset. Let $x \leadsto \rho_{x}$ be a weak* continuous map of $X$ into $\left\{\mu \in C^{*}(Y): \mu(1)=1\right\}$. Suppose $X$ is divided into three disjoint pieces $X_{1}, X_{2}$, and $X_{3}$ with the following properties:

(1) For each $x \in X_{2}, \rho_{x}$ is a probability measure.

(2) For each $x \in X, \rho_{x} \mid X_{1} \cup X_{2} \equiv 0$.

(3) For each $x \in X_{3}, \rho_{x}=\delta(x)$.

(4) $X_{1} \cup X_{2} \neq Y$.

Let $A=\left\{f \in C(Y): f(x)=\rho_{x}(f)\right.$ for all $\left.x \in X\right\}$. We note that $1 \in A$. Let $\Phi: C^{*}(Y) \rightarrow A^{*}$ be the canonical map. For a set $F \subseteq Y$, we let

(1) Partially supported by NSF grant GP-20856 A\# 1.

Received by the editors February 9, 1973.

AMS (MOS) subject classifications (1970). Primary 46E15; Secondary 46G99, 52A05, 54C35. 


$$
P(F)=\left\{\mu \in C^{*}(Y): \mu \geq 0, \mu(1)=1, \mu \text { supported by } F\right\} .
$$

Let $K=\Phi P(Y)$. We shall identify $y \in Y$ with $\Phi \delta(y) \in K$. Let $\partial$ be the Choquet boundary of $A$ which, by our identification, is a subset of $Y$. Clearly, $\partial$ $\subseteq Y-X_{2}$. We now make the further assumption:

(5) $\partial=Y-X_{2}$.

First, some obvious remarks.

Remark 1. $X_{1}, X_{2}$, and $X_{3}$ are Borel sets.

Indeed, it is trivial that $X_{3}$ is closed. As $\partial=Y-X_{2}$, we have that $X_{2} \cup X_{3}$ $=\rho^{-1}(P(Y))$ and so $X_{2} \cup X_{3}$ is closed. The remark is now clear.

Remark 2. There is an $M<\infty$ so that $\left\|\rho_{x}\right\|<M$ each $x \in X$.

Indeed, since $X$ is compact and $\rho$ is weak* continuous, the image of $\rho$ is weak* compact and, so, norm bounded.

\section{Theorem 1.}

$$
\begin{aligned}
A^{\perp}=\left\{w \in C^{*}(Y): w(B)\right. & =w\left(B \cap X_{1}\right)+w\left(B \cap X_{. .}\right) \\
& \left.-\int_{X_{1} \cup X_{2}} \rho_{x}(B) d w(x) \text { for each Borel } B \subseteq Y\right\} .
\end{aligned}
$$

Proof. The proof is contained in the proof of Theorem 1.2 of [5], which we reproduce here. We may assume $X_{3} \neq \varnothing$ with no loss of generality (if it is empty, add a point of $Y-\left(X_{1} \cup X_{2}\right)$ to $\left.X\right)$. Let

$$
M=\left\{\delta(x)-\rho_{x}: x \in X_{1} \cup X_{2}\right\}
$$

and $X^{\prime}=M \cup\{0\}=\left\{\delta(x)-\rho_{x}: x \in X\right\}$. As the map

$$
x \rightarrow \delta(x)-\rho_{x}
$$

is continuous, $X^{\prime}$ is weak compact. The map (1) is clearly one-to-one from $X_{1} \cup X_{2}$ onto $M$. Since $X_{3}$ is closed, both $M$ and $X_{1} \cup X_{2}$ are locally compact. Hence (1) is a proper map [2, Chapter $1, \S 10$, No. 3, Corollary] and so $X_{1} \cup X_{2}$ is homeomorphic to $M$ [2, Chapter $1, \S 10$, No. 1, Proposition 2].

Let $Z$ be the weak* closed convex hull of $X^{\prime}$ and $W$ its linear span. Clearly the weak* closure of $W$ is $A^{\perp}$. Using [7, Proposition 1.2], we get

$$
\begin{aligned}
& W=\left\{w \in C^{*}(Y) \text { : There is a bounded regular Borel measure } \nu^{\prime}\right. \\
& \left.\quad \text { on } M \text { such that } w(f)=\int_{M} p(f) d \nu^{\prime}(p) \text { for each } f \in C(Y)\right\} .
\end{aligned}
$$

Fix $w \in W$ and find the associated measure $\nu^{\prime}$ on $M$. Using the homeomorphism between $M$ and $X_{1} \cup X_{2}, \nu^{\prime}$ induces a measure $v$ on $X_{1} \cup X_{2}$. Passing to finite sums and taking limits we get 


$$
\begin{aligned}
w(f) & =\int_{M} p(f) d \nu^{\prime}(p) \\
& =\int_{X_{1} \cup X_{2}}\left(\delta(x)-\rho_{x}\right)(f) d \nu(x) \\
& =\nu(f)-\int_{X_{1} \cup X_{2}} \rho_{x}(f) d \nu(x) .
\end{aligned}
$$

But then we get $[3, \mathrm{~V}, \S 3$, Corollary to Proposition 12]

$$
w(B)=\nu(B)-\int_{X_{1} \cup X_{2}} \rho_{x}(B) d \nu(x)
$$

for each Borel $B \subseteq Y$. In particular, for $B \subseteq X_{1} \cup X_{2}$ we have $\rho_{x}(B)=0$ and so $w(B)=\nu(B)$. Thus $w \mid X_{1} \cup X_{2}=\nu$ and so

$W=\left\{w \in C^{*}(Y)\right.$ : For each Borel $B \subseteq Y$,

$$
\left.w(B)=w\left(B \cap X_{1}\right)+w\left(B \cap X_{2}\right)-\int_{X_{1} \cup X_{2}} \rho_{x}(B) d w(x)\right\} .
$$

Thus, to complete the proof, we need show that $W$ is already weak ${ }^{*}$ closed. By $[4$, V.5.9] it suffices to show $W$ is norm closed. But this is clear using Remark 2 above.

Recall that for a measure $\mu$ on a nonempty compact set $X$ in a lctvs $E$, $r(\mu)=x$ is the resultant of $\mu$ if for each continuous linear functional $f$ on $E$ we have $f(x)=\int f d \mu$.

Lemma 2. With the identification of $y \in Y$ with $\Phi(\delta(y))$ in $A^{*}$, we have:

(1) $K=\Phi P(\partial)$.

(2) If $x \in C^{*}(Y)$ is the resultant of $\mu$, a measure supported by $\operatorname{Ext}(K)$, then regarding $\mu$ as a measure on $Y$ supported by $\partial$ we have $\Phi(\mu)=x=r(\mu)$.

(3) Let $\mu \in C^{*}(Y)$ and associate to it a measure $\mu^{\prime}$ on $\partial$ by

$$
\mu^{\prime}(B)=\mu(B \cap \partial)+\int_{X_{2}} \rho_{x}(B) d \mu(x) .
$$

Then $r\left(\mu^{\prime}\right)=\Phi(\mu)$.

Proof. (1) is just [7, Proposition 1.2]. For (2), let $f \in A=$ weak* linear functionals on $K$. Then

$$
\begin{aligned}
x(f) & =\int_{\operatorname{Ext}(K)} p(f) d \mu(p)=\int_{\partial} \delta(x)(f) d \mu(x) \\
& =\mu(f)=\Phi(\mu)(f) .
\end{aligned}
$$

As for (3), let $f \in A$. Then

$$
\begin{aligned}
f\left(r\left(\mu^{\prime}\right)\right) & =\int_{\operatorname{Ext}(K)} p(f) d \mu^{\prime}(p)=\mu^{\prime}(f) \\
& =\int_{\partial} f(x) d \mu(x)+\int_{X_{2}} \rho_{x}(f) d \mu(x)=\int_{Y-X_{2}} f(x) d \mu(x)+\int_{X_{2}} f(x) d \mu(x)
\end{aligned}
$$


since $\rho_{x}=\delta(x)$ for $x \in X_{2}$ on $A$

$$
=\mu(f)=\Phi(\mu)(f) .
$$

A set $D \subseteq Y$ is said to be full if the following conditions hold:

(1) For all $x \in X_{1},\left(\delta(x)+\rho_{x}\right)(B) \neq 0$ for some $B \subseteq D$ implies that $\delta(x)+\rho_{x}$ is supported by $D$.

(2) For all $x \in X_{2}, x \in D$ implies $\rho_{x}$ is supported by $D$.

Elementary properties of full sets are contained in the next lemma.

Lemma 3. (1) If $D$ is full, then for all $x \in X_{1}$, we have:

$$
\begin{aligned}
\left(\delta(x)+\rho_{x}\right)(B) & \neq 0 \text { for some } B \subseteq D^{C} \\
& \Rightarrow \delta(x)+\rho_{x} \text { is supported by } D^{C} .
\end{aligned}
$$

(2) If $D$ or $D^{C}$ is full, then for all $x \in X_{1}$ we have:

$$
x \in D^{C} \Rightarrow \rho_{x} \mid D \equiv 0 .
$$

(3) $D$ is full iff the following two conditions hold:

(a) For all $x \in X_{1}, x \in D^{C} \Rightarrow \rho_{x}$ supported by $D^{C}$.

(b) For all $x \in X_{1} \cup X_{2}, x \in D \Rightarrow \rho_{x}$ supported by $D$.

Corollary 4. Let $D$ be full. Let $\mu$ be supported by $D \cap \partial$ and $\nu$ be supported by $D^{c} \cap \partial$. If $\Phi(\mu)=\Phi(\nu)$, then $\Phi(\mu)=0=\Phi(\nu)$. Hence, neither $\mu$ nor $\nu$ can be positive measures.

Proof. Since $\Phi(\mu)=\Phi(\nu)$, we have $\mu-\nu \in A^{\perp}$. Thus, for any Borel $B \subseteq Y$ we have, by Theorem 1 ,

$$
\begin{aligned}
\mu(B)-\nu(B)= & \mu\left(B \cap X_{1}\right)+\mu\left(B \cap X_{2}\right)-\int_{X_{1} \cup X_{2}} \rho_{x}(B) d \mu-\nu\left(B \cap X_{1}\right) \\
& -\nu\left(B \cap X_{2}\right)+\int_{X_{1} \cup X_{2}} \rho_{x}(B) d \nu .
\end{aligned}
$$

Let $E \subseteq D$ be Borel. Then $\nu(E)=\nu\left(E \cap X_{1}\right)=\nu\left(E \cap X_{2}\right)=0$. By Lemma $3(2), \int_{X_{1}} \rho_{x}(E) d \nu=0$. As $\nu$ is supported by $\partial=Y-X_{2}, \int_{X_{2}} \rho_{x}(E) d \nu=0$. Hence, from (2),

$$
\mu(E)=\mu\left(E \cap X_{1}\right)+\mu\left(E \cap X_{2}\right)-\int_{X_{1} \cup X_{2}} \rho_{x}(E) d \mu .
$$

Similar reasoning shows that for Borel $G \subseteq D^{C}, \mu(G)=\mu\left(G \cap X_{1}\right)=\mu(G$ $\left.\cap X_{2}\right)=\int_{X_{1} \cup X_{2}} \rho_{x}(G) d \mu=0$. Hence, (3) holds for each Borel set in $Y$ and so $\mu \in A^{\perp}$. Hence, $\Phi(\mu)=0=\Phi(\nu)$. If $\mu$ were positive, say, then $\|\mu\|=\mu(1)>0$. As $1 \in A, \mu(\mathbf{1})=\Phi(\mu)(\mathbf{1})=0$, a contradiction.

For ease of notation, for each $D \subseteq Y$ we let $T_{D}=\Phi P(D)=\left\{\Phi(\nu) \in A^{*}: \nu\right.$ is a probability measure supported by $D$ \}. 
Proposition 5. If $D$ is full, then both $T_{D}$ and $T_{\partial-D}$ are faces of $K$.

Proof. As the map $\Phi$ is affine, both $T_{D}$ and $T_{\partial-D}$ are convex subsets of $K$. Suppose $k \in T_{D}$ and $k=\alpha n+(1-\alpha) m$ with $n, m \in K$ and $0<\alpha<1$. Choose $\mu$ supported by $D \cap \partial$ and $\tau, \lambda$ in $P(\partial)$ so that $k=\Phi(\mu), n=\Phi(\tau)$, and $m=\Phi(\lambda)$. In order to show $T_{D}$ is a face, it clearly suffices to show that $\nu=\alpha \tau+(1-\alpha) \lambda$ is supported by $D \cap \partial$. Write $\nu=\nu|(D \cap \partial)+\nu|\left(D^{C} \cap \partial\right)$. Noting that $\Phi(\nu)=k=\Phi(\mu)$, we get $\Phi\left(\nu \mid\left(D^{C} \cap \partial\right)\right)=\Phi(\mu-\nu \mid(D \cap \partial))$. By Corollary $4, \nu \mid D^{C} \cap \partial \equiv 0$ as it cannot be positive. Thus $\nu \in P(D \cap \partial)$ and so $T_{D}$ is a face. A similar argument shows $T_{\partial-D}$ is a face.

We recall that for a face $F$ of $K$, the union of all faces disjoint from $F$ is denoted by $F^{\prime}$. If face $(k)$ denotes the minimal face of $K$ containing $k$, then $F^{\prime}=\bigcup\{$ face $(k): k \notin F\}$. To show that $T_{D}$ is a split face for $D$ closed and full, we must first describe $T_{D}^{\prime}$. Toward this goal, we have the following lemma.

Lemma 6. Suppose $T_{D}$ is a face of $K$. Then $T_{D}^{\prime} \subseteq T_{\partial \cap D C}$.

Proof. Let $k \in T_{D}^{\prime}$. Then $k=\Phi(\mu)$ for $\mu$ a probability measure supported by d. We claim that $\mu(D)=0$. Indeed, if not, then $\mu=\alpha(\mu \mid D) / \mu(D)+(1-\alpha) \nu$ where $\nu$ is either the zero measure or is supported by $\partial-D$. Then

$$
k=\alpha \Phi\left(\frac{\mu \mid D}{\mu(D)}\right)+(1-\alpha) \Phi(\nu)
$$

shows that $\Phi((\mu \mid D) / \mu(D)) \in$ face $(k)$. As $(\mu \mid D) / \mu(D) \in P(D)$ we have $\Phi((\mu \mid D) / \mu(D)) \in$ face $(k) \cap T_{D}=\varnothing$ since $k \in T_{D}^{\prime}$. This contradiction establishes the claim and the lemma.

Theorem 7. Let $D$ be a closed full set. Then $T_{D}$ is a closed split face of $K$ with Ext $T_{D}=D \cap \partial$ and $T_{D}^{\prime}=T_{\partial \cap D C}$.

Proof. The proof proceeds in three steps. We first claim that $T_{D}$ is a closed face. From Proposition 5, we need only show that $T_{D}=\Phi P(D)$ is compact. As $\Phi$ is continuous and $P(D)$ compact, this is clear. Also clear are the facts Ext $T_{D}$ $=D \cap \partial$ and $T_{D}=T_{D \cap \partial}$. We next claim that $T_{D}^{\prime}=T_{\partial \cap D C}$. From Lemma 6, we need only show that $T_{\partial \cap D^{c}} \subseteq T_{D}^{\prime}$. Let $n \in T_{D} \cap T_{\partial \cap D^{c}}$. Then $n=\Phi(\mu)=\Phi(\nu)$ with $\mu \in P(D \cap \partial)$ and $\nu \in P\left(D^{C} \cap \partial\right)$. But this clearly cannot occur by Corollary 4 and so $T_{D} \cap T_{\partial \cap D^{C}}=\varnothing$. As $T_{\partial \cap D^{c}}$ is a face, $T_{\partial \cap D^{c}} \subseteq T_{D}^{\prime}$.

Finally, we claim that $T_{D}$ is split. Let $k \in K-\left(T_{D} \cup T_{D}^{\prime}\right)$ and suppose we have two decompositions of $k$ :

$$
\begin{aligned}
k & =\alpha_{1} n_{1}+\left(1-\alpha_{1}\right) m_{1} \\
& =\alpha_{2} n_{2}+\left(1-\alpha_{2}\right) m_{2}
\end{aligned}
$$

where $0<\alpha_{i}<1, n_{i} \in T_{D}$, and $m_{i} \in T_{D}^{\prime}$. Find $\mu_{i} \in P(D \cap \partial)$ and $\nu_{i} \in P\left(D^{C}\right.$ $\cap \partial)$ so that $\Phi\left(\mu_{i}\right)=n_{i}$ and $\Phi\left(\nu_{i}\right)=m_{i}$. Then

$$
\Phi\left(\alpha_{1} \mu_{1}+\left(1-\alpha_{1}\right) \nu_{1}\right)=\Phi\left(\alpha_{2} \mu_{2}+\left(1-\alpha_{2}\right) \nu_{2}\right)
$$


and so

$$
\Phi\left(\alpha_{1} \mu_{1}-\alpha_{2} \mu_{2}\right)=\Phi\left(\left(1-\alpha_{2}\right) \nu_{2}-\left(1-\alpha_{1}\right) \nu_{1}\right)
$$

From Corollary 4 we get

$$
\Phi\left(\alpha_{1} \mu_{1}-\alpha_{2} \mu_{2}\right)=0=\Phi\left(\left(1-\alpha_{2}\right) \nu_{2}-\left(1-\alpha_{1}\right) \nu_{1}\right) .
$$

Applying $\alpha_{1} \mu_{1}-\alpha_{2} \mu_{2}$ to $1 \in A$ we get $\alpha_{1}-\alpha_{2}=0$. Hence (4) yields $\Phi\left(\mu_{1}-\mu_{2}\right)$ $=0$. Hence $n_{1}=n_{2}$. Similarly $m_{1}=m_{2}$ and the decompositions of $k$ coincide.

We should now like to begin to prove a converse of Theorem 7 .

Lemma 8. Let $s \in X$ and suppose $\Phi(\tau)=\Phi\left(\rho_{s}\right)$ for some measure $\tau$ supported by $\left(X_{1} \cup X_{2}\right)^{C}$. Then $\tau=\rho_{s}$.

Proof. If $s \in X_{3} \subset \partial$, then $\tau=\delta(s)=\rho_{s}$ clearly. So assume that $s \in X_{1} \cup X_{2}$. Since $\tau-\delta(s) \in A^{\perp}$, by Theorem 1 , for each Borel $B \subseteq Y$,

$$
\begin{aligned}
\tau(B)-\delta(s)(B)= & \tau\left(B \cap X_{1}\right)+\tau\left(B \cap X_{2}\right)-\int_{X_{1} \cup X_{2}} \rho_{x}(B) d \tau-\delta(s)\left(B \cap X_{1}\right) \\
& -\delta(s)\left(B \cap X_{2}\right)+\int_{X_{1} \cup X_{2}} \rho_{x}(B) d \delta(s)(x) \\
= & \tau\left(B \cap X_{1}\right)+\tau\left(B \cap X_{2}\right)-\int_{X_{1} \cup X_{2}} \rho_{x}(B) d \tau-\delta(s)(B)+\rho_{s}(B) .
\end{aligned}
$$

As $\tau$ is supported by $\left(X_{1} \cup X_{2}\right)^{C}$, we get

$$
\tau(B)-\delta(s)(B)=-\delta(s)(B)+\rho_{s}(B)
$$

and the result is immediate.

Theorem 9. Let $F$ be a closed split face of $K$. Let $D=\overline{F \cap \partial}$. Then $F=T_{D}, F^{\prime}$ $=T_{\partial \cap D c}$, and $D$ is full.

Proof. Since $F \cap \partial=$ Ext $F$, we have $F=\overline{c o}(F \cap \partial)$ and so $F=T_{F \cap \partial}$ $=T_{D}$. We claim that $F^{\prime}=T_{\partial \cap D C}$. Indeed, let $y \in \partial \cap D^{C}$. Then face $(y)=$ $\{y\}$ is disjoint from $F$ and so $y \in F^{\prime}$. Now let $\mu \in P\left(\partial \cap D^{C}\right) \subseteq P\left(F^{\prime}\right)$. Then [1, Corollary II.6.11] implies that $r(\mu) \in F^{\prime}$. Hence $T_{\partial \cap D^{c}} \subseteq F^{\prime}$. As Lemma 6 yields $F^{\prime}=T_{D}^{\prime} \subseteq T_{\partial \cap D C}$ we have proven our claim.

To show that $D$ is full, we will use the criteria of Lemma 3(3). Let $s \in X_{1} \cup X_{2}$. Let $\nu_{1}=\rho_{s}^{+}\left|D, \nu_{2}=\rho_{s}^{+}\right| \partial \cap D^{C}, \nu_{3}=\rho_{s}^{-} \mid D$, and $\nu_{4}=\rho_{s}^{-} \mid \partial \cap D^{C}$. Let $\beta_{i}$ $=\left\|\nu_{i}\right\|$ and $\mu_{i}=\nu_{i} /\left\|\nu_{i}\right\|$ (take $\mu_{i}=0$ whenever $\left\|\nu_{i}\right\|=0$ ). Then $\rho_{s}=\beta_{1} \mu_{1}$ $+\beta_{2} \mu_{2}-\beta_{3} \mu_{3}-\beta_{4} \mu_{4}$. Hence

$$
\Phi\left(\delta(s)+\beta_{3} \mu_{3}+\beta_{4} \mu_{4}\right)=\Phi\left(\beta_{1} \mu_{1}+\beta_{2} \mu_{2}\right) .
$$

Applying these elements of $A^{*}$ to $1 \in A$ we get

$$
1+\beta_{3}+\beta_{4}=\beta_{1}+\beta_{2} \text {. }
$$

Let $\beta$ be this common number. 
We first assume that $s \in F$. Then

$$
s /\left(1+\beta_{3}\right)+\beta_{3} \Phi\left(\mu_{3}\right) /\left(1+\beta_{3}\right)=f \in F
$$

and so

$$
\frac{1+\beta_{3}}{\beta} f+\frac{\beta_{4}}{\beta} \Phi\left(\mu_{4}\right)=\frac{\beta_{1}}{\beta} \Phi\left(\mu_{1}\right)+\frac{\beta_{2}}{\beta} \Phi\left(\mu_{2}\right) .
$$

From the characterization of $F^{\prime}$ we know that $\Phi\left(\mu_{2}\right), \Phi\left(\mu_{4}\right) \in F^{\prime}$. Since $f, \Phi\left(\mu_{1}\right)$ $\in F$ and $F$ is split, (6) implies that $f=\Phi\left(\mu_{1}\right), \Phi\left(\mu_{4}\right)=\Phi\left(\mu_{2}\right)$, and $1+\beta_{3}=\beta_{1}$. Plugging this into (5) yields

$$
\Phi\left(\delta(s)+\beta_{3} \mu_{3}\right)=\Phi\left(\beta_{1} \mu_{1}\right)
$$

and so $\Phi\left(\beta_{1} \mu_{1}-\beta_{3} \mu_{3}\right)=\Phi\left(\rho_{s}\right)$. Since $\beta_{1} \mu_{1}-\beta_{3} \mu_{3}$ is supported by $\left(X_{1} \cup X_{2}\right)^{C}$, Lemma 8 implies that $\beta_{1} \mu_{1}-\beta_{3} \mu_{3}=\rho_{s}$. Hence $\rho_{s}$ is supported by $D$. So we have shown that $s \in D \cap\left(X_{1} \cup X_{2}\right)$ implies $\rho_{s}$ is supported by $D$. Similarly, for $s \in D^{C} \cap X_{1} \subseteq \partial \cap D^{C} \subseteq F^{\prime}$, we get $\rho_{s}$ is supported by $\partial-D \subseteq D^{C}$. Hence all the criteria of Lemma 3(3) have been verified.

We have now arrived at our characterization of facially closed sets. Recall that a set $D \subseteq$ Ext $K$ is facially closed if there is a closed split face $F$ with Ext $F=D$.

Theorem 10. Let $D \subseteq Y-X_{2}$. Then $D$ is facially closed if and only if the following conditions hold:

(a) $D$ is closed in $Y-X_{2}$.

(b) $\bar{D}$ is full.

Proof. Suppose conditions (a) and (b) hold. From (b) and Theorem 7, we get $T_{\bar{D}}$ is a closed split face and Ext $T_{\bar{D}}=\bar{D} \cap \partial$. From (a), $\bar{D} \cap \partial=D$ and so $D$ is facially closed. Conversely, suppose $D$ is facially closed. As all facially closed sets are closed in Ext $K=\partial$, we get (a). Also, there is a closed split face $F$ with $F \cap \partial=D$. Theorem 9 now yields (b).

As an example of Theorem 10 we cite the following construction. Suppose $X$, $Y, \partial$ and $\rho$ are as above. Suppose further:

(a) $X_{2}=\varnothing$.

(b) $\rho_{s}$ is a finite linear combination of point masses in $X_{1}^{c}$ for each $s \in X_{1}$.

(c) For $s \neq s^{\prime}$ elements of $X_{1}, \operatorname{supp} \rho_{s} \cap \operatorname{supp} \rho_{s}^{\prime}=\varnothing$.

On $Y$ define an equivalence relation $\sim$ by the following: $p \sim q$ means that there exists an $s \in X_{1}$ with both $p$ and $q$ in supp $\rho_{s} \cup\{s\}$.

Theorem 11. Assume the above hold. Then the factor topology on $Y / \sim$ "is" the facial topology on $Y-X_{2}$ and it is always $T_{1}$.

Proof. A set $F \subseteq Y / \sim$ is closed iff $F$ is closed in $Y$ and $F$ is saturated for the relation $\sim$. Note that $F$ is saturated for the relation $\sim$ iff for every $s \in X_{1}$, (supp $\left.\rho_{s} \cup\{s\}\right) \cap F \neq \varnothing$ implies supp $\rho_{s} \cup\{s\} \subseteq F$. But the latter statement is 
precisely the statement that $F$ is full. Thus, by Theorem $10, F \subseteq Y / \sim$ is closed iff $F$ is facially closed in $Y$.

Example 1 (P. Taylor). Let $Y$ be the shaded region of the plane in the following diagram:

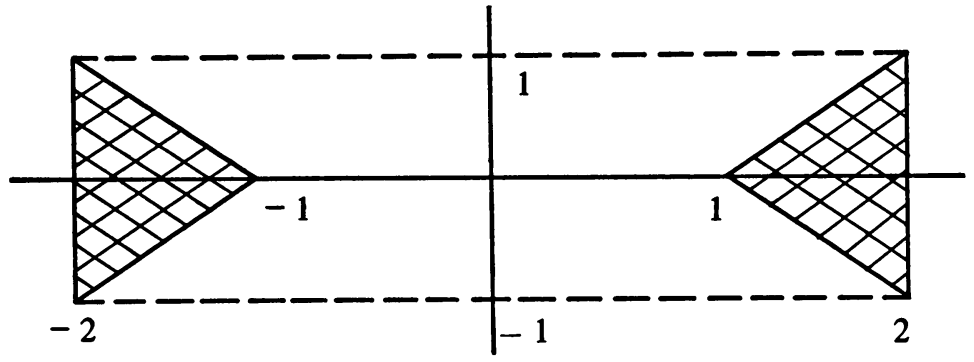

We take $X=\{(x+1, y): 0 \leq y \leq x, 0 \leq x \leq 1\}$ and define for $s \in X$ :

$$
\rho_{s}=\delta(-x-1,-y)+\delta(x+1,-y)-\delta(-x-1, y) \text {. }
$$

Clearly $s \leadsto \rho_{s}$ is weak* continuous on $X$ and $\rho_{s}(1)=1$. We have $X_{2}=\varnothing$ and $X_{3}=\{(x+1, y) \in X: y=0\}$, with $X_{1}=X-X_{3}$. Let

$$
A=\left\{f \in C(Y): f(s)=\rho_{s}(f) \text { for all } s \in X\right\} .
$$

Then clearly each $y \in Y$ is a peak point for $A$, i.e. for all $y \in Y$, there is an $f \in A$ with $f(y)=1>|f(p)|$ for $p \neq y$. Hence $\partial=Y$ and so all five of our assumptions are fulfilled. We define $\sim$ on $Y$ as we did above: $p \sim q$ iff there is an $s \in X$ with $p, q \in \operatorname{supp} \rho_{s} \cup\{s\}$. Clearly each element of the decomposition under $\sim$ is of the form $\{(x+1, y),(x+1,-y),(-x-1, y),(-x-1,-y)\}$ for $0 \leq y \leq x, 0 \leq x \leq 1$. It is easily verified that the projection map $\pi: Y \rightarrow Y / \sim$ is open so the factor space $Y / \sim$ with the factor topology is first countable, second countable, compact, and locally compact though not Hausdorff. Via Theorem 11, the facial topology on $Y$ is first countable, second countable, compact, and locally compact though not Hausdorff. In [6], we studied the facial topology (among other topologies on the extreme points of compact convex sets). We proved there that if $(Y$, facial topology) satisfied an auxiliary condition $(\mathrm{C} 2)$, then the properties of first countability, second countability, and local compactness for ( $Y$, facial topology) were equivalent. We did not know then whether $(\mathrm{C} 2)$ was necessary for the conclusion. The above construction provides the necessary example for it does not satisfy even $(\mathrm{Cl})$, a weaker condition than (C2).

Proposition 12. There is a compact metrizable convex set $K$ with closed extreme points whose facial topology is first countable, second countable and locally compact but which does not satisfy:

(C1) If $\left\{p_{n}\right\} \subseteq \operatorname{Ext}(K)$ converges to $q$ and if $\left\{p_{n}\right\}$ converges to $p$ in the facial topology, then $p$ belongs to the minimal closed split face containing $q$. 
Proof. We take $K$ to be the set $\left\{\mu \in A^{*}: \mu(1)=1=\|\mu\|\right\}$. Then $\operatorname{Ext}(K)=Y$. We take $p_{n}=(1+1 / n, 1 / n), q=(1,0)$, and $p=(-1,0)$. Clearly $p_{n} \rightarrow q$ and $\left\{p_{n}\right\}$ converges to both $p$ and $q$ in the facial topology. Finally, the minimal closed split face containing $q$ is $\{q\}$ which clearly does not contain $p$.

Example 2. (Rogalski in [8]). Let $d x$ be Lebesgue measure on $[0,1]$ and $\mu$ be the measure $\sum_{n=1}^{\infty} 2^{-n} \delta\left(x_{n}\right)$ where $\left\{x_{n}\right\}$ is an enumeration of the rational numbers in $[0,1]$. We take $X_{1}=X_{3}=\varnothing, X_{2}=\left\{x_{1}\right\}$ and

$$
\rho_{x_{1}}=2 d x-2 \sum_{n=2}^{\infty} 2^{-n} \delta\left(x_{n}\right) \text {. }
$$

Then

$$
\begin{aligned}
A & =\left\{f \in C[0,1] \mid f(x)=\rho_{x}(f) \text { all } x \in X\right\} \\
& =\left\{f \in C[0,1] \mid \sum 2^{-n} f\left(x_{n}\right)=\int f d x\right\} .
\end{aligned}
$$

All the assumptions regarding the map $\rho$ are trivial in this case and [8, Proposition 20] shows that $\partial=[0,1]$ so all of our results above apply. Thus, by Theorem 10 , a set $D \subseteq[0,1]$ is facially closed iff $D$ is a closed subset of the irrational numbers in $[0,1]$ or $D=[0,1]$. Hence, each irrational number in $[0,1]$ is a split face (a fact already established in [8, Corollary 25]) but no rational number in $[0,1]$ is a split face.

Proposition 13. There exists a compact convex set $K$ whose extreme points $E$ form a closed set for which the collection of extreme points which are split faces are dense in $E$ and for which the collection of extreme points which are not split faces are dense in $E$.

\section{REFERENCES}

1. E. Alfsen, Compact convex sets and boundary integrals, Springer-Verlag, New York and Berlin, 1971.

2. N. Bourbaki, General topology. Part 1, Addison-Wesley, Reading, Mass., 1966. MR 34 \# 5044b.

3 - Eléments de mathématique. Fasc. XXI. Livre V: Intégration, 2ieme ed., Actualities Sci. Indust., no. 1244, Hermann, Paris, 1967. MR 35 \#322.

4. N. Dunford and J. Schwartz, Linear operators. I. General theory, Pure and Appl. Math., vol. 7, Interscience, New York, 1958. MR 22 \#8302.

5. A. Gleit, On the existence of simplex spaces, Israel J. Math. 9 (1971), 199-209. MR 43 \#5293.

6. - Topologies on the extreme points of compact convex sets, Math. Scand. 31 (1972), 209-219.

7. R. Phelps, Lectures on Choquet's theorem, Van Nostrand, Princeton, N. J., 1966. MR 33 \# 1690.

8. M. Rogalski, Characterisation des simplexes par des propriétés portant sur les faces fermées et sur les ensembles compacts de points extremaux, Math. Scand. 28 (1971), 159-181.

Department of Mathematics, University of Massachusetts, amherst, Massachusetts 01002 